\title{
Caracterización de la Artritis reumatoidea en el consultorio de especialidades del ISSS
}

\author{
Rubén Antonio Montúfar Guardado \\ José Alberto Rodríguez Hernández ${ }^{2}$ \\ Yolanda Rodríguez de Romero ${ }^{3}$ \\ Raúl Antonio Aragón Laínez ${ }^{4}$ \\ Claudia Castellanos de Bará5
}

\section{RESUMEN}

a Artritis Reumatoidea (AR) es una enfermedad inflamatoria crónica, poliarticular e incapacitante. En la Consulta de Reumatología del Consultorio de Especialidades del Instituto Salvadoreño del Seguro Social (ISSS), es una de las principales causas de consulta y es responsable de muchas discapacidades y muertes prematuras. El objetivo del estudio fue describir las principales características demográficas, clínicas y de laboratorio de una muestra de pacientes con AR que son atendidos en la Consulta. El tipo de estudio fue observacional, descriptivo, y de corte transversal. Se incluyeron 686 pacientes con diagnóstico de AR. El 89.2 fueron mujeres, con edad promedio de 50.2 (21 - 87) años. El nivel de educación fue similar en ambos sexos, encontrando un $3.4 \%$ de analfabetismo. El $45.3 \%$ de los pacientes referidos por primera vez a la consulta, tenía menos de 3 años de evolución de su enfermedad, y el 21.3\% tenía más de 10 años de evolución, lo cual se encontraba muy relacionado con el grado mayor de discapacidad (EL 9.7\% se encontraba en clase funcional III o IV). El 62.2\% tenían el factor reumatoide positivo. En conclusión, y en similitud a lo reportado, encontramos que la Artritis Reumatoidea es una enfermedad que afecta al $89.2 \%$ de las mujeres, con edades promedio de 50.2 años. Alrededor de la mitad de los pacientes (45.3\%) son referidos oportunamente a la Consulta de Reumatología, lo cual se ve reflejado en un menor grado de discapacidad.

Palabras clave: Artritis Reumatoide, características clínicas, aspectos demográficos, El Salvador. 


\section{ARTÍCULOS ORIGINALES}

\section{INTRODUCCIÓN}

La Artritis Reumatoidea (AR) es una enfermedad inflamatoria crónica, poliarticular y simétrica, que afecta principalmente las articulaciones diartrodiales. Se caracteriza por causar daño estructural importante en la membrana sinovial, cartilago articular y hueso, el cual se ve reflejado en discapacidad funcional progresiva y muerte prematura. ${ }^{1,2,3}$

Es una enfermedad de distribución mundial, de etiología desconocida, que afecta aproximadamente al $1.2 \%$ de la población en general. Es más común en mujeres, con una relación hombre mujer de 3:1 y se presenta con una mayor frecuencia entre la cuarta y sexta década de la vida. 4,5 Muchos pacientes son incapaces de trabajar después de 10 años de evolución de la enfermedad y arriba de un 80\% de las personas se encuentran incapacitados después de 20 años de evolución de padecerla. La esperanza de vida se reduce del 3 al 18\% en la población que la padece. ${ }^{6}$ A nivel de Latinoamérica, son muy pocos los estudios que demuestran la presentación de la enfermedad en la zona, siendo el Grupo Latinoamericano de Estudio de Artritis Reumatoide (CLADAR), la principal cohorte mejor representada en la región. En ese estudio, se incluyeron 1093 pacientes, de 14 países, encontrando que el $85 \%$ eran mujeres, con edades promedio al inicio de su enfermedad de $46 \pm 14.2$ años, y con un alto grado de actividad (Disease Activity Score $($ DAS 28$)=5.9 \pm 1.6)$ y discapacidad secundario a la enfermedad (Health Assessment Questionnaire $(H A Q)=1.4 \pm 0.8)^{7}$. El Salvador es un país con una población estimada de 5.7 millones, en donde la mayor cobertura en salud la brinda el Ministerio de Salud Pública y Asistencia Social (MINSAL); por otra parte el Instituto Salvadoreño del Seguro Social únicamente atiende al $23 \%$ de la población. El Consultorio de Especialidades es el principal centro de referencia de consulta ambulatoria especializada de la Seguridad Social, brindando consultas de tercer nivel a 123,000 derecho habientes en el año, correspondiendo el 8\% de ellas (12,345 consultas) a los pacientes de Reumatología. ${ }^{8}$

En El Salvador, son muy pocos los estudios publicados que muestran las características de los pacientes con enfermedades reumáticas, y es por ello que el objetivo del presente estudio fue describir las principales características demográficas, clínicas y de laboratorio de una muestra de pacientes con AR, Es importante mencionar, que los pacientes con Artritis Reumatoidea constituyen la consulta más frecuente en la Consulta de Reumatología en el ISSS ${ }^{9}$

\section{MATERIALES Y MÉTODOS}

En el período comprendido de Octubre a Diciembre del 2009, se realizó un estudio observacional, descriptivo, prospectivo y de corte transversal. Se incluyeron 686 pacientes con AR, según los criterios establecidos por el Colegio Americano de Reumatología y los cuales fueron elegidos al azar. La recolección de datos se realizó mediante un cuestionario en el cual se incluían aspectos demográficos, clínicos y de laboratorio. La información fue tabulada y analizada mediante el programa SPSS 15.0, utilizando promedios y desviaciones estándar para las variables continuas de números y porcentajes para las variables categóricas.

\section{RESULTADOS}

El 89.2\% ( $n=616$ ) de los pacientes fueron mujeres. La edad promedio encontrada fue de 50.2 años (21 - 87) en el grupo de las mujeres y de 54.4 años (20 - 81) en el grupo de los hombres. En la gran mayoría de los pacientes en ambos grupos la enfermedad inició entre los 40 y los 60 años. En relación a la procedencia, el 83.1\% provenían de la zona central, el $12.5 \%$ del occidente y el $4.4 \%$ de la zona oriental del país. De los referidos por primera vez, el 45.3\% tenía menos de 3 años de evolución, el 33.4 $\%$ de 3 a 10 años, y un 21.3\% más de 10 años. En cuanto al nivel de educación, fue similar en ambos 
sexos, encontrando que el $3.4 \%$ eran analfabetos, el $16 \%$ había finalizado su bachillerato y un $27 \%$ había completado sus estudios universitarios (Tabla 1 ).

Tabla 1. Aspectos demográficos de la población

\begin{tabular}{|c|c|}
\hline Caracteristicas & $n(\%)$ \\
\hline $\begin{array}{l}\text { - Sexo } \\
\text { Hombre } \\
\text { Mujer }\end{array}$ & $\begin{array}{l}70(10.8) \\
616(89.2)\end{array}$ \\
\hline $\begin{array}{l}\text { - Edad media en } \\
\text { hombres ( rango ) }\end{array}$ & $54.4(20-81)$ \\
\hline $\begin{array}{l}\text { - Edad media en } \\
\text { mujeres ( rango ) }\end{array}$ & $50.2(21-87)$ \\
\hline $\begin{array}{l}\text { - Nivel de estudios: } \\
\text { Ninguno } \\
\text { Menos de } 6 \text { años de } \\
\text { estudio } \\
\text { Primarios } \\
\text { Secundarios } \\
\text { Bachillerato } \\
\text { Superiores }\end{array}$ & $\begin{array}{c}\% \\
3.4 \\
13.6 \\
2 \\
9 \\
16 \\
38\end{array}$ \\
\hline $\begin{array}{l}\text { - Lugar de procedencia } \\
\text { Zona occidental } \\
\text { Zona central } \\
\text { Zona oriental }\end{array}$ & $\begin{array}{l}12.5 \% \\
83.1 \% \\
4.4 \%\end{array}$ \\
\hline $\begin{array}{l}\text { - Evolución de la } \\
\text { enfermedad: } \\
\text { De o a } 5 \text { años } \\
\text { De } 5 \text { a } 9 \text { años } \\
\text { De } 10 \text { o más años }\end{array}$ & $\begin{array}{l}44.7 \% \\
14.4 \% \\
40.9 \%\end{array}$ \\
\hline
\end{tabular}

La capacidad funcional es un factor condicionante del pronóstico a largo plazo, sin embargo, en el grupo de pacientes estudiados, la mayoría se encontró en Clase Funcional I (55.5\%), lo cual implica que el daño ocasionado por la enfermedad es mínimo y que el paciente es capaz de llevar a cabo su vida normalmente. La clase funcional II se da en el paciente que ya tiene cierto daño, pero que aun asi es capaz de desempeñar una actividad laboral sin mayor dificultad, en este grupo se encontraba el $34.8 \%$. El 9.7\% se encontraba en clase funcional III o IV y eran aquellos que tenían una larga evolución de su enfermedad (Figura 1).

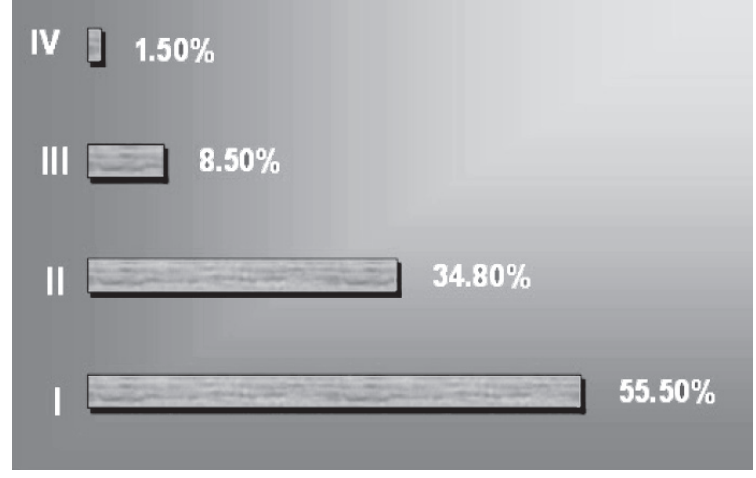

Figura 1. Clase funcional de Artritis Reumatoide

En el $4.5 \%$ había sido necesario la colocación de prótesis, siendo la más frecuente a nivel de las rodi\|las (78\%).

En cuanto a los exámenes de laboratorio, se encontró que el 62.2\% tenía el factor reumatoide positivo. El resto no mostraban alteración, excepto cierto grado de anemia principalmente en aquellos que se encontraban activos de su enfermedad. La principal causa de actividad de la enfermedad fue la suspensión de medicamentos.

En relación al tratamiento, se encontró que el 65.4 \% utilizaba esteroides en dosis promedio de $6.9 \mathrm{mg} / \mathrm{dia}$. El inmunosupresor más utilizado fue el metotrexate, un $62.5 \%$ de los pacientes lo utilizaba al momento del estudio (Figura 2).

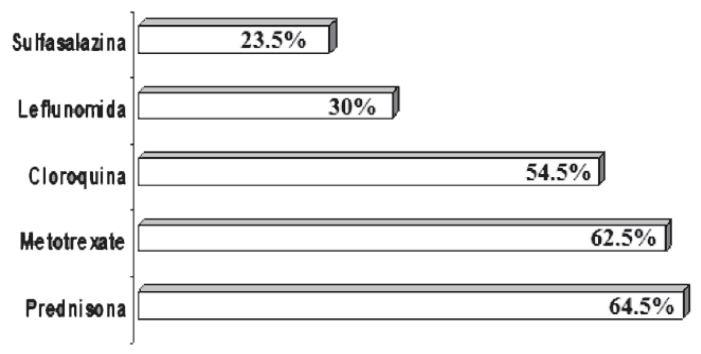

Figura 2. Tratamientos utilizados en pacientes con Artritis Reumatoide 
El inmunosupresor mas utilizado como monoterapia fue el metotrexate, seguido de cloroquina y leflunomide . La doble terapia más frecuente fue la de cloroquina con metotrexate (34.8\%). Únicamente el 9\% estaba siendo tratado con triple terapia (Tabla 2).

Tabla 2. Monoterapia o terapia combinada en Artritis Reumatoide

\begin{tabular}{|l|l|}
\hline Medicamentos & Porcentaje \\
\hline Metotrexate & 62.5 \\
\hline Metotrexate + Cloroquina & 34.8 \\
\hline Metotrexate + Cloroquina + Leflunomida & 9 \\
\hline
\end{tabular}

Ninguno de los pacientes incluidos en el estudio se encontraba utilizando medicamentos biotecnológicos. Un 7 \% fue considerado refractario al tratamiento convencional.

\section{DISCUSIÓN}

La Artritis Reumatoide es la patología más frecuentemente atendida en la consulta ambulatoria de Reumatología en el Consultorio de Especialidades. A nivel mundial, la prevalencia de Artritis Reumatoide oscila entre $0.3-1.2 \%{ }^{10}$, sin embargo según los estudios de Camboa y Cardiel, la prevalencia de Artritis es más baja en Latinoamérica, oscilando entre el $0.3-0.5 \%{ }^{11,12}$

De manera similar a lo reportado en la literatura, en El Salvador son las mujeres las más frecuentemente afectadas, pero con una relación Mujeres/Hombres de 9.1. La edad promedio de los pacientes en estudio (52.8 años), es ligeramente más baja a la reportada en diferentes estudios descriptivos, quienes encuentran edades promedios que oscilan entre los 56 a los 61 años..$^{13,14}$ El bajo nivel de educación formal, es un factor demográfico condicionante de mal pronóstico en pacientes con AR; sin embargo, en los pacientes estudiados se encontró un 3.2\% de analfabetismo, pero solo un $27 \%$ había finalizado sus estudios universitarios. Otro dato importante a considerar, es el hecho de que casi la mitad de los pacientes con artritis (45.4\%) fueron referidos tempranamente a nuestra consulta (menos de 3 años de evolución), lo cual se ve reflejado en un porcentaje bajo de pacientes con discapacidad funcional importante (9.8\% de los pacientes en clase funcional
III y IV). Este dato, es bastante similar a lo reportado en Chile por Massardo et al ${ }^{15}$, quienes encuentran un $18 \%$ de los pacientes en clase III o IV y contrasta de manera importante con lo reportado por Sany et al ${ }^{16}$ quienes encuentran más de la mitad de los pacientes en clase funcional III o IV (55\%). El porcentaje de pacientes sometidos a cirugía de reemplazo articular es bajo (4.5\%) cuando se compara con lo reportado por Sany et al, quienes encuentran que un $47.8 \%$ había sido sometido a cirugía de reemplazo articular ${ }^{16}$.El $62.2 \%$ tenían el factor reumatoide positivo, dato muy similar al descrito en la mayoría de estudios a nivel mundial. ${ }^{17}$

En cuanto a tratamiento, un $65.4 \%$ se encontraba en el momento del estudio con esteroides en dosis bajas ( $6.9 \mathrm{mg} / \mathrm{dl}$ ), el cual estaba siendo utilizado de manera permanente en algunos pacientes y en otros únicamente como terapia de puente. Estos datos son muy parecidos a lo reportado por Sany et al (72\% de los pacientes con esteroides $)^{16}$ y al encontrado en CLADAR. (77\% de los pacientes con prednisona en dosis menores a $10 \mathrm{mg} / \mathrm{dí})^{17}$.

El Metotrexate fue el inmunosupresor más utilizado, lo cual concuerda con lo reportado en la mayoría de estudios clínicos. Un 62.5\% se encontraba con un FARME como monoterapia, 34.8\% con doble terapia y un $9 \%$ con triple terapia. La doble terapia más utilizada fue la combinación de metotrexate más cloroquina. Este dato concuerda con lo reportado en Francia en cuanto a monoterapia, pero en doble o triple terapia el porcentaje es notablemente menor en ese estudio.

\section{CONCLUSIONES}

La Artritis Reumatoidea es una enfermedad frecuente, que afecta principalmente a mujeres. Es evidente que un diagnóstico temprano y un tratamiento adecuado y oportuno cambia de manera considerable el pronóstico de la enfermedad, evitando las 
discapacidades, co morbilidades y prolongando la sobrevida de los pacientes.

\section{REFERENCIAS BIBLIOCRÁFICAS}

1. Lee David M. Rheumatoid arthritis. Lancet 2001; 358:90311

2. Harris E D. Rheumatoid arthritis: Pathophysiology and implications for therapy. New England Journal of Medicine 1990: 322:1277-89

3. Lawrence R C, Helmick C C, Arnett F C, et al. Estimates of the prevalence of arthritis and selected musculoskeletal disorder in the Unites States. Arthritis Rheum 1998;41:778-99

4. Cabriel SE. The epidemiology of rheumatoid arthritis. Rheum Dis Clin North Am 2001;27:269-81

5. Symmons $D$ P, Barret E M, Bankhead CR, et al. The incidence of rheumatoid arthritis in the United Kingdom: results from the Norfolk Arthritis Register. Br. Journal of Rheumatology 1994;33:735-9

6. Doran M F., Pond C R, Crowson C S et al. Trends in incidence and mortality in arthritis rheumatoid in Rochester MN. Arthritis an Rheum 2001 ; 44: 1276, S265

7. GLADAR (Grupo Latinoamericano de Arrititis Reumatoide). Demographics and Clinical Characteristics of Early Rheumatoid Arthritis in an Inception Cohort of Latin American Patients. Journal of Clinical Rheumatology 2006:12:4 S 41

8. Anuario Estadístico del Instituto Salvadoreño del Seguro Social. Subdirección Administrativa. Dirección de Desarrollo y Planificación Administrativa. Abril 2010. http://wnwisss. gob.sv/
9. Montúfar R, Castillejos G, Rodriguez Y, Rodriguez J, Aragón $\mathrm{R}$ and Castellanos C. Epidemiological profile of theumatology outpatient clinics in El Salvador. Journal of Clinical Rheumatology 2012;18;9: S32

10. Sherine $C$ and Kaleb Michaud. Epidemiological studies in incidence, prevalence, mortality, and comorbidity of the rheumatic diseases. Arthritis Research \& Therapy 2009, 11:229: 1-16

11. Camboa R, Medina M, Acevedo E y cols. Prevalencia de enfermedades reumatológicas y discapacidad en una comunidad urbano marginal: resultados del primer estudio COPCORD en el Perú. Revista Peruana de Reumatología 2009;15;1:40-46

12. Cardiel M and Rojas J. Community based study to estimate prevalence, burden of illness and help seeking behavior in rheumatic disease in Mexico City. A COPCORD study. Clin Exp Rheum 2002:20:617-24

13. Carmona L, Conzalez-Alvaro I, Balsa A, Angel Belmonte M, Tena $X$ and Sanmarti R. Rheumatoid arthritis in Spain: Occurrence of extra-articular manifestations and estimates of disease severity. Ann Rheum Dis 2003;62:897-900.

14. Leardini G, Salaffi F, Montanelli R, Gerzeli S, Canesi B. A multicenter cost-of - illness study on rheumatoid arthritis in Italy. Clinical and Experimental Rheumatology 2002;20:505-15.

15. Massardo L, Aguirre V, Carcía ME, Cervilla V, Nicovani S, Conzález A et al. Clinical expression of rheumatoid arthritis in Chilean patients. Semin Arthritis Rheum 1995; 25: 203-13

16. Sany J, Bourgeois P, Saraux A and cols. Characteristics of patients with rheumatoid arthritis in France: a study of 1109 patients managed by hospital based rheumatologist. Ann Rheum Dis 2004; 63: 1235-1240

17. Mody Girish M. and Cardiel Mario H. Challenges in the management of rheumatoid arthritis in developing countries. Best Practice \& Research Clinical Rheumatology 2008; Vol 22, No 4, pp 621-641. 